\title{
COMUNICACIÓN
}

\section{Pesca con cebo activo por Ardea alba (Pelecaniformes: Ardeidae) en Argentina}

\author{
Lorena Vanesa Sovrano ${ }^{1,2}$ \& Adolfo Héctor Beltzer ${ }^{1}$ \\ 1. Instituto Nacional de Limnología (CONICET - UNL) Paraje El Pozo s/n, Ciudad Universitaria, 3000-Santa Fe, Argentina. Facultad de Ciencia \\ y Tecnología. \\ 2. Universidad Autónoma de Entre Ríos, Km 10,5 RP11, Oro Verde, 3100-Entre Ríos, Argentina; lorenavsovrano@hotmail.com
}

Recibido 17-VI-2017 • Corregido 29-VIII-2017 • Aceptado 11-IX-2017

\begin{abstract}
Active bait-fishing by Ardea alba (Pelecaniformes: Ardeidae) in Argentina. The behavior of active bait fishing has been recorded in species of the family Ardeidae. For the Great Egret (Ardea $a l b a$ ) there is only one previous record in the literature. By this contribution, a new record of active bait fishing in a Urban Park by the Great Egret is reported.
\end{abstract}

Keywords: Ardeidae, tool-use, Argentina, feeding
RESUMEN: El comportamiento de pesca con cebo activo ha sido registrado en especies de la familia Ardeidae. Para la Garza blanca (Ardea alba) sólo existe un registro previo en la literatura. Mediante este aporte se documenta el registro de pesca con cebo activo por la Garza blanca observado en un Parque urbano de Argentina.

Palabras claves: Ardeidae, uso de herramientas, Argentina, alimentación
La Garza blanca (Ardea alba) es una especie con amplia distribución en América, encontrándose en ríos, lagunas, arroyos, esteros, pantanos, entre otros ambientes relacionados con el agua (De la Peña, 2015). Su alimentación consiste en animales capturados en el agua o en sus proximidades. La dieta está compuesta fundamentalmente por peces, insectos (larva y adultos), anfibios y en menor cantidad por crustáceos, arácnidos y restos de vegetales (Beltzer \& Oliveros, 1981; De la Peña \& Salvador, 2010; Lorenzón, Ronchi Virgolini \& Beltzer, 2012). Esta especie permanece quieta en la orilla, o se desplaza lentamente en busca de presas que pasan a su alcance y cuando llega el momento, utiliza su pico como un arpón y las atrapa (Olivares, 1973).

Al tratarse de una especie cosmopolita, el estudio de su ecología trófica puede servir de base para comprender la plasticidad de la especie, o por el contario, la similitud entre diferentes ecosistemas acuáticos naturales o antrópicos (Lorenzón et al., 2012).

Un ave que manipula y coloca pequeños objetos comestibles (cebo) o materiales no comestibles (señuelo) en la superficie del agua cercanos al pescador (Higuchi, 1986) para atraer o distraer a posibles presas, exhibe un comportamiento particular llamado pesca de cebo activo (Ruxton \& Hansell, 2011) o baiting (Kushlan \& Hancock, 2005), que se clasifica como una forma de uso de herramientas (Crain, Giray \& Abramson, 2013). Se puede diferenciar la pesca con cebo activo que implica la identificación y colocación del mismo (pan, insectos) en el agua para atraer peces u otros animales acuáticos, y la pasiva, donde el pescador se siente atraído por un cebo y espera para capturar la presa (Gavin \& Salomon, 2009).

Este comportamiento ha sido reportado en especies de la familia Ardeidae. La mayoría de las observaciones 
se han hecho en Butorides sp. (Lovell, 1958; Keenan, 1981; Walsh, Grunewald \& Grunewald, 1985; Preston, 1986; Higuchi, 1988a, 1988b; Oake, 1992; Robinson, 1994; McCoullough \& Beasley, 1996) y en Nycticorax nycticorax (McCoullough \& Beasley, 1996; Gavin \& Solomon, 2009; Riehl, 2001; Réglade, Siel \& Uribe, 2014). Para Ardea alba existe un primer registro de Lovell (1958) describiendo un comportamiento de pesca pasiva puesto que el ave, sin manipular el cebo, se ubicó cerca de trozos de pan depositados en el agua para atrapar los peces que eran atraídos por los mismos. Recientemente, se registró por primera vez la pesca con cebo activo en esta especie (Réglade, dos Santos \& Mitchell, 2015). La mayoría estas observaciones han sido en ambientes artificiales o modificados y utilizando como cebo materiales provenientes de los seres humanos (pan). Estudios de comportamiento que involucra el uso de herramientas, son importantes para entender el desarrollo cognitivo de las aves y las innovaciones que presentan (Kushlan, 2011). Desde este enfoque, el objetivo de este trabajo fue documentar un registro de pesca con cebo activo por Ardea alba, incorporando información sobre este comportamiento.
La observación se registró en el Parque Ecológico Municipal José Gazzano (31 46 $80^{\prime \prime}-31^{\circ} 46^{\prime} 75^{\prime \prime} \mathrm{N}$ y $60^{\circ} 30^{\prime} 96^{\prime \prime}-60^{\circ} 30^{\prime} 03^{\prime \prime} \mathrm{W}$ ) en la ciudad de Paraná (Entre Ríos, Argentina). El Parque es un Paisaje Protegido con 8ha de extensión, que posee una laguna artificial, zonas de recreación e importantes arboleadas. Este espacio verde se ubica en una matriz urbana que ha crecido rápidamente en los últimos años (Sovrano, 2016).

Las observaciones se realizaron desde enero hasta marzo de 2015 y suman un total de aproximadamente 80 horas. Mediante estas observaciones se registró el forrajeo de un individuo de Ardea alba desde dos puntos fijos cercanos a las orillas de la laguna, a ojo descubierto o con binoculares, documentando el comportamiento mediante fotografías. Cuando el animal pescó utilizando cebo se registró: el tipo de cebo, el éxito de pesca y el tiempo aproximado que duró la actividad.

Ardea alba utilizó migas de pan como cebo. Este elemento es arrojado por los visitantes para alimentar a las aves domésticas del parque (Anser anser, Cairina moschata). La garza se ubicó en las orillas de la laguna donde observó las piezas flotantes de pan, las recogió y las colocó cercanas a su ubicación. Permanecía inmóvil con una

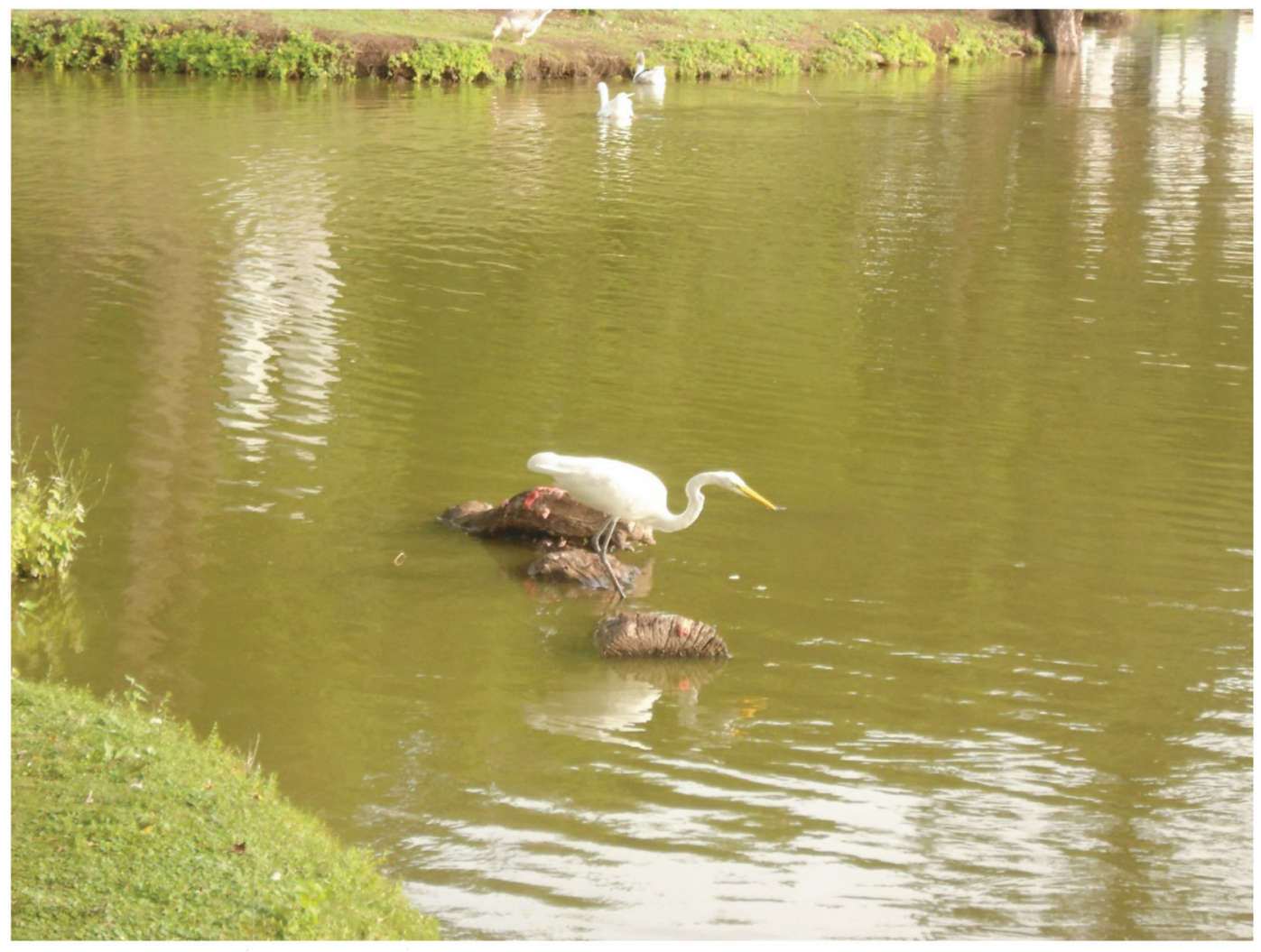

Fig.1. Garza blanca (Ardea alba) capturando una presa mediante la pesca con cebo activo en el Parque Ecológico Municipal José Gazzano de Paraná, Entre Ríos (Argentina). 
postura inclinada prestando atención a los movimientos en el agua. En reiteradas ocasiones pretendió capturar los peces, cuando no tuvo éxito, retiró el cebo y lo colocó nuevamente en la superficie, utilizando el mismo punto de alimentación. Esta secuencia se repitió durante 30 a 60 minutos aproximadamente. Las presas capturadas fueron peces de pequeño tamaño (Fig. 1).

Para otras especies de la familia Ardeidae se ha identificado el uso de una gran variedad de cebos (pan, galletas, insectos, alimento de peces, lombrices) o señuelos (ramas, materiales de plástico, plumas; Higuchi, 1986, 1988a; Ruxton \& Hansell, 2011). En el caso de la Garza blanca, ya se ha registrado previamente el uso de porciones de pan (Lovell, 1958; Réglade, dos Santos et al., 2015). Las observaciones se han realizado en parques urbanos, sitios en los que los individuos tienen disponible cebos tales como trozos de pan. Posiblemente, la Garza blanca aprendió este comportamiento observando a los seres humanos alimentar a las aves domésticas, asociando los trozos de pan con la aparición de los peces, como ha sido sugerido por otros autores (Pratt, Denis, Walther \& Walther, 2011; Réglade, dos Santos, et al., 2015). Además, el cebo de pesca utilizado proviene de un elemento utilizado por el hombre. El uso de objetos naturales ha sido descripto como un comportamiento más evolucionado, que hasta el momento no ha sido registrado en A. alba (Higuchi, 1986). Algunos autores sugieren que la pesca pasiva es antecesora a la activa (Gavin \& Salomon, 2011), lo que hace llamativo que no se hayan documentado casos de pesca pasiva en $A$. alba, teniendo en cuenta además que se trata de una especie cosmopolita, ampliamente distribuida en América, África y Australia (Lorenzón et al., 2012).

La Garza blanca exhibió un comportamiento de pesca con cebo activo, similar a lo informado en el trabajo de Réglade, dos Santos et al.(2015). El animal manejó la porción de pan según su éxito de captura y aparentemente tuvo más resultados efectivos al utilizar el cebo (cinco capturas en 20 minutos utilizando cebo vs dos en el mismo tiempo sin utilizar cebo). No obstante, otros factores pueden contribuir en esta aparente ventaja de la pesca con cebo, como el tiempo de pesca, el grado de saciedad y la disponibilidad de cebos.

Este trabajo incorpora un nuevo registro de pesca con cebo activo, ampliando los conocimientos de este comportamiento poco frecuente, probablemente debido a restricciones cognitivas o ecológicas (Réglade, Dilawar \& Anand, 2015), registrado en 11 especies de garzas (Réglade, dos Santos et al., 2015). Además, de modo más general, contribuye al conocimiento del uso de herramientas y la innovación de técnicas de captura dentro de las aves (Overington, Morand-Ferron, Boogert \& Lefebvre, 2009).

\section{REFERENCIAS}

Beltzer, A. H., \& Oliveros, O. B. (1981). Alimentación de aves en el valle aluvial del río Paraná Medio II. Egretta alba egretta (Gmelin, 1789) y Egretta thula thula (Molina, 1782) (Ciconiiformes: Ardeidae). Ecología Argentina, 6, 119-124.

Crain, B. J., Giray, T., \& Abramson, C. I. (2013). A tool for every job: assessing the need for a universal definition of tool use. The Internacional Journal of Comparative Psychology, 26, 281-303.

De la Peña, M. R., \& Salvador, S. A. (2010). Manual de la alimentación de las aves argentinas. Santa Fe: Ediciones UNL.

De la Peña, M. R. (2015). Aves Argentinas: huevos y nidos. Buenos Aires, Argentina: Eudeba- Ediciones UNL.

Gavin, M. C., \& Salomon, J. N. (2009). Active and Passive bait-fishing by Black-Crowned Night Herons. The Wilson Journal of Ornithology, 121(4), 844-845. doi: 10.1676/09-049.1

Higuchi, H. (1986). Bait- fishing by the Green- Backed Heron Ardeola striata in Japan. Ibis, 128, 285-290. doi: 10.1111/j.1474-919X.1986.tb02677.x

Higuchi, H. (1988a). Bait-fishing by Green-Backed Herons in South Florida. Field Naturalist, 16, 8-9.

Higuchi, H. (1988b). Individual differences in bait-fishing by the Green-Backed Heron Ardeola striata associated with territory quality. Ibis, 130, 39-44. doi: 10.1111/j.1474919X.1988.tb00953.x

Keenan, W. J. III (1981). Green heron fishing with mayflies. Chat, 45,41 .

Kushlan, J. A., \& Hancock, J. A. (2005). The Herons. New York: Oxford Univ. Press.

Kushlan, J. A. (2011). The terminology of courtship, nesting, feeding and maintenance in herons. Recuperado de http:// www.heronconservation.org/resources/Behavior_ Terminology.pdf

Lorenzón, R. E., Ronchi Virgolini, A. L., \& Beltzer, A. H. (2012). Ecología trófica de la Garza blanca Ardea alba (Pelecaniformes: Ardeidae) en un humedal del río Paraná, Argentina. UNED Research Journal, 5(1), 121- 127.

Lovell, H. B. (1958). Baiting of fish by a Green Heron. Wilson Bulletin, 70, 280-281.

McCoullough, D. D., \& Beasley, R. J. (1996). Bait fishing herons: have some birds learned to use tools. Bird Watchers Digest, 18, 49-59.

Oake, K. (1992). More bait-fishing by Green Backed Heron in Botswana. Babbler, 23, 49. 
Olivares, A. (1973). Las Ciconiiformes colombianas. Bogotá, Colombia: Proyser Tercer Mundo.

Overington, S. E., Morand-Ferron, J., Boogert, N. J., \& Lefebvre, L. (2009). Technical innovations drive the relationship between innovativeness and residual brain size in birds. Animal Behavior, 78, 1001-1010. doi: 10.1016/j. anbehav.2009.06.033

Pratt, H. D., Denis, J., Walther, M., \& Walther, C. (2011). Rapid and widespread appearance of bait-fishing a form of tool use, by Black-crowned Night Herons in Hawaii. Elepaio, 71 (4), 24-28.

Preston, C. R. (1986). Green-Backed Heron baits fish with insects. Wilson Bulletin, 98, 613- 614.

Réglade, M. A., Siel, D., \& Uribe, M. (2014). Bait fishing Blackcrowned Night Heron (Nycticorax nycticorax) in Chile. Ornitología Neotropical, 25, 465-468.

Réglade, M. A., dos Santos, A. C., \& Mitchell, K. (2015a). First records of active bait fishing for Great (White) Egrets (Ardea alba). Journal of Heron Biology and Conservation, 12, 2. Recuperado de http://www.heronconservation.
org/wp-content/uploads/2015/08/12-Reglade_et_al_ white_egret1.pdf

Réglade M. A., Dilawar, M. E., \& Anand, U. (2015b). Active bait-fishing in Indian Pond Heron Ardeola grayii. Indian Birds, $10(5), 124-125$

Riehl, C. (2001). Black-crowned Night Heron fishes with bait. Waterbirds, 24, 285- 286. doi: 10.2307/1522044

Robinson, S. K. (1994). Use of bait and lures by Green Backed Herons in Amazonian Perú. Wilson Bulletin, 106, 567-569.

Ruxton, G. D., \& Hansell, M. H. (2011). Fishing with a Bait or Lure: A Brief Review of the Cognitive. Ethology, 117, 1-9. doi: 10.1111/j.1439-0310.2010.01848.x

Sovrano, L. V. (2016). Riqueza de aves en el Parque Ecológico Municipal José Gazzano (Paraná, Entre Ríos, Argentina). $F A B I C I B, 20,123-132$.

Walsh, J. F., Grunewald, J., \& Grunewald, B. (1985). GreenBacked Herons (Butorides striatus) possibly using a lure and using apparent bait. Journal of Ornithology, 126(4), 439- 442. doi: 10.1007/BF01643409 